Digital Press Social Sciences and Humanities

Le contexte culturel de la langue maternelle dans la production écrite en langue étrangère des étudiants de I'Universitas Negeri Semarang

Neli Purwani

Proceeding of Conférence internationale sur le français 2018

Joesana Tjahjani, Merry Andriani, Sajarwa, Wening Udasmoro (eds) 


\title{
Le contexte culturel de la langue maternelle dans la production écrite en langue étrangère des étudiants de l’Universitas Negeri Semarang
}

\author{
Neli Purwani \\ Universitas Negeri Semarang, Semarang, Indonesia \\ e-mail : npurwani@mail.unnes.ac.id
}

\section{Résumé}

Dans le cours de la production écrite, l'enseignant donne des tâches pour que les étudiants pratiquent leur compétence. Les contextes culturels des tâches présentées dans des méthodes FLE sont parfois loin de l'imagination de l'apprenant. Pour réduire le problème de la xénité culturelle, il est nécessaire pour un professeur de modifier ces taches pour pouvoir encourager l'apprenant à l'activité de production. Pour produire des textes qui représentent le niveau avancé, l'étudiant doit être dirigé pour pouvoir s'exprimer des idées de manière logique. Dans ce contexte, l'approche cognitive semble convenable à appliquer dans le cours de la production écrite. Cet article détaille l'adaptation des taches de la production écrite du contexte culturel de la langue française à celui de la langue de l'apprenant et aussi de décrire l'application de l'approche cognitive dans l'apprentissage de la compétence de la production écrite au niveau avancé.

\section{Mots-clés}

adaptation, approche cognitive, production écrite, tâche

\begin{abstract}
In the course of the written production, the teacher gives tasks for the students to practice their skill. The cultural contexts of tasks presented in FLE methods are sometimes far from the learner's imagination. To reduce the problem of cultural xenicity, it is necessary for a teacher to modify these spots in order to encourage the learner to produce. To produce texts that represent the advanced level, the student must be directed in order to express ideas logically. In this context, the cognitive approach seems appropriate to apply in the course of written production. This article details the adaptation of the tasks of the written production of the cultural context of the French language to that of the language of the learner and also describes the application of the cognitive approach in the learning of the competence of the written production at the advanced level.
\end{abstract}

\section{Keywords}

adaptation, cognitive approach, written production, task

\section{Introduction}

L'apprenant d'une langue étrangère a déjà des connaissances linguistiques de sa langue première. Cette langue est aussi connue comme la langue maternelle, la langue premièrement acquise par un individu (Castelotti, 2001, p. 22). L'une des rôles de la langue maternelle est comme une langue de référence dans l'acquisition des savoirs et des savoirs faire de la langue étrangère. Dans la pratique communicative, la langue maternelle est utilisée dans la préparation des énoncés, parce que le travail cognitif d'un individu se réalise en langue qui est déjà internalisée chez lui. C'est donc une langue proche. Une langue qui montre la façon de penser d'un individu.

Dans l'apprentissage du français comme une langue étrangère à l'UNNES, des méthodes du français langue étrangère publiées par des maisons d'éditions française sont utilisé comme une source, et aussi 
comme le fil conducteur. Ces livres présentent des textes, des exercices et des taches qui mènent l'apprenant à l'acquisition de la langue. Les textes, les exercices, et les taches sont présentés dans le contexte culturel de la langue cible. Ce contexte culturel de la langue cible est parfois difficile à comprendre pour un apprenant parce qu'il est loin de l'imagination de l'apprenant. L'apprenant face donc à deux types de la xénité de la langue étrangère : celle de la langue et celle de la culture.

La xénité de contexte culturel pourrait empêcher l'acquisition de cette langue étrangère de l'apprenant, c'est pourquoi il est nécessaire, en tant qu'un enseignant, d'adapter le contexte culturel de la langue cible à celui de la langue de l'apprenant surtout dans la partie des taches.

Des taches à un rôle important dans l'apprentissage de langue étrangère. Un apprenant peut pratiquer la langue à travers des taches. Les taches dans l'apprentissage de la langue étrangère sont des exercices qui permettent l'apprenant à utiliser la langue. De point de vue de la didactique de langue, la tâche est la clé de l'approche actionnelle. Par des taches, "un apprenant mobilise l'ensemble de ses compétences et de ses ressources pour parvenir au résultat qu'il escompte : la réussite de la communication langagière". (Tagliante, 2005, p. 36) Autrement dit, elles sont des exercices par lesquelles un apprenant réalise des projets pour montrer ses compétences.

Les caractéristiques des taches dans l'apprentissage de langue étrangère sont variées selon le niveau de l'apprenant. Dans le cas l'apprentissage du français au niveau avancé, elles sont préparées pour rendre l'apprenant plus indépendant à s'exprimer et communiquer les idées, en le permettant de s'habituer à bien organiser ses idées. Dans ce contexte, l'approche cognitive semble convenable à appliquer dans le processus de l'apprentissage.

Cette approche semble convenable pour le cours de la production écrite au niveau avancé, car dans ce cours, les apprenants doivent être capables de mettre en œuvre tous les principes linguistiques pour communiquer des idées par des textes écrits qui examinent un thème / sujet de manière large et approfondie, en utilisant des mots appropriés au contexte. Pour pouvoir atteindre à cette compétence, l'apprenant devrait penser à son texte ; comment est-ce qu'il va développer son texte et organiser ses idées, quels mots qu'il va utiliser, quels principes de grammaire qu'il va appliquer pour tel ou tel phrase. Ces activités sont des activités cognitives où il produit des mots et des phrases de manière logique.

\section{Objectifs de l'étude}

Cette étude a pour objectif de décrire l'adaptation des taches de la production écrite du contexte culturel de la langue française à celui de la langue de l'apprenant et aussi de décrire l'application de l'approche cognitive dans l'apprentissage de la compétence de la production écrite au niveau avancé. Dans un premier temps, nous nous intéresserons à la forme de l'adaptation des taches de la production écrite du contexte culturel français au contexte culturel dont l'apprenant a déjà maîtrisé.

Dans un second temps, nous nous intéresserons au processus de l'application de l'approche cognitive dans l'apprentissage de la compétence de la production écrite et l'importance de cette approche à l'apprentissage de la langue étrangère au niveau avancé.

\section{Méthode}

Le public de cette étude est des apprenants au niveau avancé dans la classe de l'observation, le cours de la production écrite avancé à l'Universitas Negeri Semarang (UNNES). Dans le cours, l'enseignant utilise la méthode de FLE de la maison d'édition «Maison des Langues », Version Originale 3. Une étude de documentation est faite pour connaître 1es taches qui sont adaptés du contexte culturel français au contexte culturel de l'apprenant. Des observations sont réalisées pour obtenir des données sur le processus du cours de la production écrite au niveau avancé avec l'approche cognitive. Les observations couvrent trois activités : l'activité initiale, activité principale, et l'activité finale. 


\section{Résultats}

\subsection{Des taches en production écrite}

L'adaptation faite pour les taches de production écrite est une adaptation de taches présentées dans le livre Version Originale 3. L'enseignant a fait l'adaptation en 3 tâches de production écrite.

La première adaptation est faite pour l'unité 2 du livre, le thème présenté est les vacances, dont l'objectif de l'apprentissage est que l'apprenant pourra proposer un voyage pour une personne. La tâche de production écrite proposée dans le livre est la rédaction d'un dépliant pour présenter une activité insolite à faire pendant les vacances dans une ville ou une région. Pour celle-ci, l'enseignant a modifié la tâche en demandant aux étudiants de rédiger une lettre à inviter un/e ami/e à passer des vacances ensemble, en leur obligeant de détailler les activités proposer, le logement, le transport et le prix.

La deuxième adaptation est faite pour l'unité 5 du livre, les thèmes présentés sont les courriers et les mobilisations pour une cause sociale. L'objectif de l'apprentissage est que l'apprenant pourra rédiger un appel à une mobilisation sociale et écrire une lettre ouverte. La tâche de production écrite proposée dans le livre est de raconter l'expérience de participer à une mobilisation. Pour cet unité, l'enseignant a demandé aux étudiant de raconter une expérience de participer à une mobilisation sociale, ou d'écrire une lettre ouverte pour inviter quelqu'un à joindre à une organisation/ mobilisation sociale.

La troisième adaptation est faite pour unité 8 , dont les thèmes sont des nouvelles et la presse. L'objectif de l'apprentissage est que l'apprenant pourra présenter un flash d'information en rapportant les paroles d'autrui. La tâche de production écrite proposée dans le livre est la rédaction d'un récit de l'aventure à la manière d'un fait divers. Pour cette tâche, l'enseignant a fait une modification en demandant aux étudiants de réécrire en français une nouvelle prise d'un journal en ligne indonésien en forme du fait divers.

\subsection{Le processus de l'application de l'approche cognitive}

Comme ce qui a été expliqué dans la partie précédente, la description du processus du cours de la production écrite au niveau avancé couvre trois activités : l'activité initiale, principale, et finale.

Premièrement, à l'activité initiale, l'étudiant est invité à comprendre les textes déclencheurs afin de comprendre le contexte situationnel de la compétence. Dans l'activité de compréhension, l'étudiant apprend le vocabulaire du contexte et connaître le phénomène présenté, comme exemple il découvert des mobilisations pour une cause sociale de la France. Ensuite, il est invité de trouver le phénomène similaire du contexte de l'apprenant.

La deuxième, l'étape principale ou l'étape de rédaction. L'enseignant donne des consignes pour guider l'étudiant. Il est conseillé d'organiser l'ordre de ses idées de manière logique. Pendant l'organisation de l'ordre des idées, l'apprenant est autorisé d'utiliser sa langue maternelle afin qu'il puisse montrer les relations logiques entre des idées. L'usage de cette langue est considéré pouvoir aider l'apprenant de relier des idées de manière logique et rationnel car c'est la langue par laquelle l'apprenant s'habitue à penser.

Après la rédaction, c'est l'étape finale ou l'étape de la correction. L'enseignant invite tous les étudiants dans la classe à étudier le texte. Le texte est présenté devant la classe à l'aide du projecteur installé dans la classe. L'enseignant et les étudiants comprennent le texte et donnent des remarques aux éléments linguistiques et à l'organisation des idées.

A partir du texte écrit, les corrections apportées aux phrases peuvent être classées comme correction et affinement de la grammaire, correction du choix des mots, correction de la logique des phrases, qui peuvent affecter le choix des mots.

La table suivante présente un exemple de texte produit par l'apprenant.

\section{Donnée}

...il y avait une grande inondation à Mijen, Demak. Donc, tous les membres du département de la sociale doivent suivre la mobilisation sociale. Cette inondation était à cause de la rivière qui s'est rompu, alors les maisons des habitants sont noyées. Les habitants devaient installer à l'évacuation. Ils ne pouvaient pas manger, garder ses santés, dormir bien. Ils ont été perdus de ses richesses. Ses élevages sont morts. Ils n'avaient rien. Il y avait beaucoup de volontaires pour les aider. Par exemple les volontaires de médicales. Ils prenaient en main les problèmes de médicale. Les 
volontaires sociales comme mes amis et moi pour aider une cause de sociale. Là-bas, je faisais de la cuisine comme des noodles, des légumes, des poissons, etc.

Ce texte de l'étudiant montre qu'il a eu des idées à communiquer par la tache donnée. Bien qu'il a fait beaucoup d'erreurs grammaticaux, il en a organisé en ordre logique. Des remarques données pour ce texte sont détaillé comme suit :

\section{Donnée No 1.}

"Cette inondation était à cause de la rivière qui s'est rompu".

Dans cette phrase, la correction est donnée pour que la structure de la phrase soit plus correcte et pour que l'idée de la phrase soit plus logique. L'idée que" la rivière qui s'est rompue" n'est pas logique, parce que dans la vérité c'est la digue de la rivière qui s'est rompue à cause du haut débit de l'eau. Voici la phrase en version corrigé "Cette inondation était causé par la digue de rivière qui s'est rompue".

\section{Donnée № 2.}

"alors les maisons des habitants sont noyées".

Pour cette proposition subordonnée, la correction est donnée en changeant la conjonction et en accordant le choix du mode du verbe. Voici la proposition en version corrigé "alors que les maisons des habitants soient noyées".

\section{Donnée $N^{\circ} 3$.}

"Ils ont été perdus de ses richesses".

Pour la donnée $\mathrm{N}^{\circ}$ 3, la correction est donnée sur le choix des mots "ses richesses", qui sont changés par les mots "leurs biens". Ces mots sont les mots qui conviennent avec le contexte.

\section{Donnée No 4.}

"Ses élevages sont morts".

Dans cette phrase, la correction est donnée sur les choix des mots et le choix du temp du verbe. Voici la phrase en version corrigé "Leurs bétails étaient morts noyés"

\section{Donnée No 5.}

"Il y avait beaucoup de volontaires pour les aider".

Dans cette phrase, la correction est donnée à l'usage de la préposition "pour". La correction sur cette donnée a aussi pour but de faire les étudiants se souvenir à l'usage du participe présente marquant une relation relative. Voici la phrase en version corrigé "Il y avait beaucoup de volontaires venant les aider.

\section{Données No 5 et No 6.}

"les volontaires de médicales", et "Ils prenaient en main les problèmes de médicale".

Pour ces deux données, la correction est donnée sur les choix des mots en remplaçant le mot "médical" en mots "la santé". Voici la version corrigée : "les volontaires de l'expert de la santé" et "Ils prenaient en main les problèmes de la santé". 


\section{Donnée $N^{\circ} 7$}

"Les volontaires sociales comme mes amis et moi pour aider une cause de sociale"

La phrase de la donnée No 7 n'a pas de verbe conjugué, la correction est donnée donc pour que la phrase soit bien structurée. La correction est aussi donnée sur le choix des mots « aider une cause de sociale ». Voici la version corrigée : «Les volontaires sociales comme mes amis et moi étions là pour prendre en main le problème social ».

\section{Donnée No 8}

"Là-bas, je faisais de la cuisine comme des noodles, des légumes, des poissons, etc."

Pour cette phrase, la remarque est faite sur le choix de mot "noodle" qui est en mot anglais. Comme la correction, le mot "noodle" est remplacé par le mot "nouilles".

\subsection{Discussion}

La correction est effectuée pour perfectionner le texte des étudiants et aussi comme une activité de récomprendre le matériel linguistique qu'ils ont appris depuis le début de l'apprentissage du français jusqu'au niveau avancé. Cette activité de ré-compréhension est également une activité visant à renforcer les connaissances linguistiques.

Les corrections apportées au texte produit par l'étudiant peuvent être définies comme une correction et un affinement syntaxique qui a pour but d'obtenir des phrases plus compréhensibles. Ces corrections sont classées en correction de la grammaire ou morphosyntaxe, correction du choix du mot, correction de la logique de l'idée qui affecte le choix des mots.

Dans la correction de la logique de la phrase, les étudiants sont invités à utiliser la langue maternelle. L'intention de l'usage de cette langue a pour but de découvrir la relation illogique des idées, de la structure des phrases, ou des choix des mots. Pour que, à la fin, ils puissent produire des phrases plus logiques. Comme dans la donnée numéro No 1, la phrase produite est « Cette inondation était à cause de la rivière qui s'est rompu". Cette phrase est équivalente avec une phrase indonésienne "Banjir tersebut disebabkan oleh sungai yang jebol ». Du point de vue de l'acceptabilité des idées, une rivière ne peut pas se rompre. C'est la digue de la rivière qui peut se rompre à cause du haut débit de l'eau. La correction de la phrase est donc basée sur un examen de la relation logique des idées dans la phrase par la méthode de traduction / en utilisant la langue maternelle.

Les activités de correction permettent également aux élèves de rappeler les règles de la grammaire des phrases qui appliquent les conjugaisons de verbes en mode subjonctif. Sur la base de la discussion en classe, les étudiants réapprennent des règles de la grammaire qu'ils ont oubliées, par exemple : le subjonctif qui est appliqué au verbe de la proposition subordonnée précédée par la conjonction "alors que". Dans cette section, l'enseignant explique à nouveau la règle de l'usage du mode subjonctif. Cette activité de correction est également une étape de médiation où les étudiants reflètent où se souviennent les connaissances initiales stockées et les remémorassent. Par cette activité, il est possible que les étudiants aient des connaissances solides qui conviennent avec leur niveau et qui peuvent soutenir leurs compétences.

\section{Conclusion}

L'adaptation des tâches de la méthode du FLE au contexte culturel de l'apprenant permet à l'enseignant de donner des tâches dont le contexte est proche à l'imagination de l'étudiant. Avec un contexte qui a un caractère proche, l'étudiant pourra avoir des idées à exprimer. Il aura un esprit optimiste de remplir cette tâche. Autrement dit, ces tâches modifiées peuvent encourager les étudiants à montrer leurs compétences.

L'application de l'approche cognitive dans le cours de production écrite au niveau avancé permet les étudiants à produire des phrases en prêtant attention aux éléments linguistiques et aux éléments de communication sous forme de messages logiques et cohérents. Les thèmes de texte entrainé à ce niveau exigent que les étudiants soient capables d'appliquer des règles de grammaire complexes, de sélectionner les mots appropriés, de décrire les idées de manière cohérente et logique. Pour que l'on puisse dire que les besoins de communication dans le contexte de l'apprentissage des langues étrangères à ce niveau sont plus critiques. Les apprenants à ce niveau peuvent également être amenés à utiliser leurs connaissances 
initiales pendant le processus qui couvre trois activités : initiale (compréhension du texte déclencheur), principal (la production du texte), et finale (la correction).

\section{Références}

Castelotti, V. (2001). La langue maternelle en classe de la langue étrangère. Millau : CLE International.

Tagliante, C. (2005). L'évaluation et le cadre européen commun. Paris: CLE International. 